\title{
Assessment of left and right ventricular functional parameters using dynamic dual-tracer $\left[{ }^{13} \mathrm{~N}\right] \mathrm{NH} 3$ and $\left[{ }^{18} \mathrm{~F}\right] \mathrm{FDG}$ PET/MRI
}

\author{
Sazan Rasul, PhD, ${ }^{a}$ Dietrich Beitzke, MD, ${ }^{\mathrm{b}}$ Tim Wollenweber, MD, ${ }^{\mathrm{a}}$ Ivo Rausch, \\ $\mathrm{PhD},{ }^{\mathrm{c}}$ Martin Lyngby Lassen, PhD, ${ }^{\mathrm{c}, \mathrm{d}}$ Marie Elisabeth Stelzmüller, MD, ${ }^{\mathrm{e}}$ \\ Markus Mitterhauser, PhD, a,f Verena Pichler, PhD, ${ }^{\text {a }}$ Thomas Beyer, PhD, ${ }^{c}$ \\ Christian Loewe, $M D,^{b}$ and Marcus Hacker, $M^{a}$ \\ a Division of Nuclear Medicine, Department of Biomedical Imaging and Image-guided Therapy, \\ Medical University of Vienna, Vienna, Austria \\ b Division of Cardiovascular and Interventional Radiology, Department of Biomedical Imaging \\ and Image-guided Therapy, Medical University of Vienna, Vienna, Austria \\ c QIMP Team, Center for Medical Physics and Biomedical Engineering, Medical University of \\ Vienna, Vienna, Austria \\ d Artificial Intelligence in Medicine Program, Cedars-Sinai Medical Center, Los Angeles, \\ California \\ e Clinical Division of General Surgery, Medical University of Vienna, Vienna, Austria \\ f Ludwig Boltzmann Institute Applied Diagnostics, Vienna, Austria
}

Received May 5, 2020; accepted Sep 16, 2020

doi: $10.1007 / \mathrm{s} 12350-020-02391-y$

Background. Cardiac positron emission tomography/magnetic resonance imaging (PET/ MRI) can assess various cardiovascular diseases. In this study, we intra-individually compared right (RV) and left ventricular (LV) parameters obtained from dual-tracer PET/MRI scan.

Methods. In 22 patients with coronary heart disease $\left(69 \pm 9\right.$ years) dynamic $\left[{ }^{13} \mathrm{~N}^{-\mathrm{NH}_{3}}\left(\mathrm{NH}_{3}\right)\right.$ and $\left[{ }^{18}\right.$ F]FDG (FDG) PET scans were acquired. The first 2 minutes were used to calculate $L V$ and RV first-pass ejection fraction (FPEF). Additionally, LV end-systolic (LVESV) and enddiastolic (LVEDV) volume and ejection fraction (LVEF) were calculated from the early (EP) and late-myocardial phases (LP). MRI served as a reference.

Results. RVFPEF and LVFPEF from FDG and $\mathrm{NH}_{3}$ as well as RVEF and LVEF from MRI were $(28 \pm 11 \%, 32 \pm 15 \%),(32 \pm 11 \%, 41 \pm 14 \%)$ and $(42 \pm 16 \%, 45 \pm 19 \%)$, respectively. LVESV, LVEDV and LVEF from EP FDG and $\mathrm{NH}_{3}$ in 8 and 16 gates were [71 (15 to $\left.213 \mathrm{~mL}\right)$, $98(16$ to $241 \mathrm{~mL}), 32 \pm 17 \%$ ] and [ $50(17$ to $206 \mathrm{~mL}), 93(13$ to $219 \mathrm{~mL}), 36 \pm 17 \%$ ] as well as [60 $(19$ to $360 \mathrm{~mL}), 109(56$ to $384 \mathrm{~mL}), 41 \pm 22 \%$ ] and [54 (16 to $371 \mathrm{~mL}), 116(57$ to $431 \mathrm{~mL}), 46 \pm$ 24\% ], respectively. Moreover, LVESV, LVEDV and LVEF acquired from LP FDG and $\mathrm{NH}_{3}$ were $(85 \pm 63 \mathrm{~mL}, 138 \pm 63 \mathrm{~mL}, 47 \pm 19 \%)$ and $(79 \pm 56 \mathrm{~mL}, 137 \pm 63 \mathrm{~mL}, 47 \pm 20 \%)$, respectively. The LVESV, LVEDV from MRI were $93 \pm 66 \mathrm{~mL}$ and $153 \pm 71 \mathrm{~mL}$, respectively.

\footnotetext{
Electronic supplementary material The online version of this article (https://doi.org/10.1007/s12350-020-02391-y) contains supplementary material, which is available to authorized users.

This article includes a PowerPoint file that should be made available as ESM on SpringerLink. Please include the standard Springer ESM text in the note/footer on the first article page. Below this, include the following text: "The authors of this article have provided a PowerPoint file, available for download at SpringerLink, which summarizes the contents of the paper and is free for re-use at meetings and presentations. Search for the article DOI on SpringerLink.com.
}

The authors have also provided an audio summary of the article, which is available to download as ESM, or to listen to via the JNC/ASNC Podcast.

Funding The study was partly funded by Siemens Healthineers, Erlangen, Germany.

Reprint requests: Marcus Hacker, MD, Division of Nuclear Medicine, Department of Biomedical Imaging and Image-guided Therapy, Medical University of Vienna, Waehringer Guertel 18-20, Floor 5L, 1090 Vienna, Austria; marcus.hacker@meduniwien.ac.at $1071-3581 / \$ 34.00$

Copyright (C) 2020 The Author(s) 
Significant correlations were observed for RVFPEF and LVFPEF between FDG and MRI $(R=$ $.51, P=.01 ; R=.64, P=.001)$, respectively. LVESV, LVEDV, and LVEF revealed moderate to strong correlations to MRI when they acquired from EP FDG and $\mathrm{NH}_{3}$ in 16 gates (all $R>.7, P$ $=.000$ ). Similarly, all $\mathrm{LV}$ parameters from LP FDG and $\mathrm{NH}_{3}$ correlated good to strongly positive with MRI (all $R>.7$, and $P<.001$ ), except EDV from NH3 weakly correlated to EDV of MRI $(R=.54, P<.05)$. Generally, Bland-Altman plots showed good agreements between PET and MRI.

Conclusions. Deriving $\mathrm{LV}$ and $\mathrm{RV}$ functional values from various phases of dynamic $\mathrm{NH}_{3}$ and FDG PET is feasible. These results could open a new perspective for further clinical applications of the PET examinations. (J Nucl Cardiol 2022;29:1003-17.) disease

Key Words: Cardiac PET/MRI • dynamic PET $\cdot{ }^{18}$ F-FDG $\cdot{ }^{13} \mathrm{~N}-\mathrm{NH} 3 \cdot$ coronary artery

\begin{tabular}{|ll|}
\hline \multicolumn{2}{|l|}{ Abbreviations } \\
CHD & Coronary heart disease \\
CMR & Cardiac magnetic resonance imaging \\
ESV & End-systolic volume \\
EDV & End-diastolic volume \\
EP & Early-phase \\
FDG & {$\left[{ }^{18} \mathrm{~F}\right] \mathrm{FDG}$} \\
FPEF & First-pass ejection fraction \\
LP & Late-phase \\
LV & Left ventricle \\
LVEF & Left ventricular ejection fraction \\
$\mathrm{NH}_{3}$ & $\left.{ }^{13} \mathrm{~N}\right] \mathrm{NH}_{3}$ \\
$\mathrm{PET}$ & Positron emission tomography \\
PET/MRI & Positron emission tomography/mag- \\
& netic resonance imaging \\
ROI & Region of interest \\
RV & Right ventricle \\
VOI & Volume of interest \\
\hline \hline
\end{tabular}

See related editorial, pp. 1018-1020

\section{INTRODUCTION}

Cardiovascular diseases, especially coronary heart disease (CHD), remain the leading cause of death worldwide. ${ }^{1}$ Besides an optimal physical examination, various non-invasive methods such as electrocardiography, echocardiography, computed tomography angiography, cardiac magnetic resonance imaging (CMR), and cardiac positron emission tomography (PET) using different radioactive tracers are nowadays available helping accurate detection and precise determination of the underlying cardiac illnesses.

The recent development of an integrated PET/MRI imaging system could further facilitate the early diagnosis of plenty cardiovascular conditions, which might consequently improve therapy management and reduce mortality among affected patients. ${ }^{2}$ Due to its excellent spatial and temporal resolution and its ability to characterize myocardial composition, CMR represents a well-established imaging method in ischemic heart disease and cardiomyopathies. CMR is considered the gold standard for assessing myocardial function and can quantify myocardial scar burden in ischemic heart disease. In addition, $\left[{ }^{13} \mathrm{~N}\right] \mathrm{NH}_{3}\left(\mathrm{NH}_{3}\right)$ PET is often used to estimate myocardial perfusion, while the role of cardiac $\left[{ }^{18} \mathrm{~F}\right]$ fluorodeoxyglucose (FDG) PET is mostly viability assessment, myocardial inflammation and tumor imaging. In patients with ischemic heart disease, the combination of these two tracers enables the identification of hibernating myocardium by identifying regions with perfusion-metabolism mismatch. ${ }^{3}$

On the other side, whole-body PET examinations using FDG are widely performed for oncological purpose like preoperative staging, characterization of suspicious tissues and lesions and response evaluation after receiving chemo- and/or immunotherapies. ${ }^{4}$ Although the availability of anticancer therapies has dramatically improved the survival rate of patients with different malignancies, these treatments are often associated with cardiotoxicities and subsequent serious cardiovascular adverse effects, mostly through congestive heart failure and left ventricular systolic dysfunction. ${ }^{5}$ Actually, due to the excellent soft tissue contrast of MRI in combination with molecular and metabolic PET data, the combined PET/MRI examination with FDG is increasingly used in oncology. ${ }^{6}$ Therefore, simultaneous evaluation of oncological status and cardiac function of these patients while performing a whole-body FDG PET/MRI scan might recognize patients at risk who still do not clinically demonstrate cardiotoxic effects of antineoplastic drugs.

In this context, various preclinical and human studies have demonstrated the feasibility of determining LV parameters using FDG PET and showed excellent correlations between left ventricular volume and function in static scans in humans. ${ }^{7,8}$ However, while the feasibility to derive RV and LV functional parameters from early dynamic PET scans was demonstrated in rodents, ${ }^{9}$ such studies with FDG PET in humans are missing or very limited. ${ }^{10,11}$ 
In the present study, we aimed to conduct an intraindividual comparison of the cardiac function and volume parameters of the right $(\mathrm{RV})$ and left $(\mathrm{LV})$ ventricle derived from different phases of dynamic FDG and $\mathrm{NH}_{3}$ PET scans. As integrated PET/MRI systems enable the simultaneous measurement of both PET and MRI, a simultaneous intra-individual comparison with respective CMR parameters as a reference was feasible.

\section{PATIENTS AND METHODS}

\section{Patients}

Integrated cardiac PET/MRI (3-Tesla, Biograph mMR; Siemens Healthcare, Erlangen Germany) in head-first supine position was performed in patients with coronary heart disease and ischemic cardiomyopathy, who were referred to our department for assessing myocardial perfusion and viability with a dual-tracer $\mathrm{NH}_{3}$ and FDG protocol. Only proper and completely performed cardiac dual-tracer PET/MRI studies were included in this retrospective analysis. Prior to the conduction of the scan, all patients have undersigned an informed consent for the examination. The study has been approved by the Ethics Committee of the Medical University of Vienna (EK: 1832/2016).

\section{Protocol for Dual-Tracer PET/CMR Examination}

The cardiac PET examinations for ECG-gated $\mathrm{NH}_{3}$ and FDG as well as the CMR examination acquired with the integrated PET/MRI followed well-established protocols that are applied for cardiac PET and CMR. ${ }^{12,13}$ All patients underwent blood glucose monitoring and received an oral $50 \mathrm{~g}$ glucose solution (glucoral, Germania Pharmazeutika, AUT) 1 hour before the FDG PET scan. Only diabetics with fasting blood glucose above $160 \mathrm{mg} / \mathrm{dL}$ did not receive the solution and obtained an intravenous bolus injection of a shortacting insulin followed by continuous monitoring of their blood glucose. Moreover, all patients received 250 mg of Acipimox (Olbetam ${ }^{\circledR}$ ) orally 2 hours before the examination; this leads to an excellent image quality and ensures an optimal myocardial FDG uptake similar to the insulin clamping technique, as previously reported by Knuuti et al. study. ${ }^{14}$ Accordingly, the FDG images showed a homogeneous distribution in all patients. The PET protocol composed of a 20 minutes dynamic $\mathrm{NH}_{3}$ scan followed by a 40 minutes dynamic FDG scan at rest in list-mode acquisition, triggered with 16 gates, and during free breathing. Both NH3 and FDG were administered intravenously as a bolus.

The simultaneously acquired CMR protocol included steady state free precession cine sequences in 2, 3, and 4 chamber view, short axis view, left ventricular outflow tract (LVOT) for evaluation of ventricular function as well as late gadolinium enhancement using phase-sensitive inversion recovery (PSIR) sequences 10 minutes after injection of $.15 \mathrm{~mL} / \mathrm{kg}$ of gadolinium (Gadovist ${ }^{\circ}$ ), as all previously described in detail. ${ }^{15,16}$ All PET/CMR scans were performed at rest only.

\section{First-Pass Ejection Fraction of Right and Left Ventricle from FDG and $\mathrm{NH}_{3}$}

To determine RV and LV first-pass ejection fraction (FPEF) from FDG and $\mathrm{NH}_{3}$ dynamic PET images, the first 2 minutes of each PET list-mode dataset were reconstructed by dividing them into 15 frames to visualize the time of first-pass of the tracer in the ventricles and creating count-rate plots over the entire heart. From this information, the proper start points and time duration of the first-pass in both ventricles was defined. Gated reconstruction of the first-pass was performed with vendor-based software (e7tools; Siemens Healthineers, Knoxville, USA) using ECG information for dividing the heart cycle into 8 gates. As reconstruction algorithm an ordinary-Poisson ordered-subsets-expectation-maximization (OP-OSEM) with point spread function resolution modeling, three iterations and 24 subsets was used. Data were corrected for attenuation, scatter, normalization, in frame decay, deadtime, and random. The reconstruction included a 4 mm FWHM Gaussian smoothing of the final images. In addition, a further $19 \mathrm{~mm}$ FWHM Gaussian smoothing was applied after reconstruction to achieve a smooth representation of the activity distribution within the ventricles.

Volumes-of-interest were placed around the RV and $\mathrm{LV}$ in the EDV, in general overestimating the true volume of the respective ventricle but trying to match with the cardiac valves. This was done by manual delineation slice-by-slice in the EDV frame by clinically experienced cardiac nuclear medicine specialists using the software Hermes Hybrid Viewer PDR, version 4.0.0 (HERMES Medical Solutions, Stockholm, Sweden) and merging the individual ROIs to the final VOIs for the $\mathrm{RV}$ and LV. 
Under the assumption that the activity in the myocardium and the background can be neglected compared to the activity in the ventricles during the FP, the total activity within these VOIs corresponds to the total activity within the respective ventricles. Therefore, the total activity in the VOIs were exported in the EDV and ESV gate to obtain the total activity $\left(\mathrm{A}_{\text {total }}\right)$ in the respective ventricle in both, the ED and ES phase. Under the assumption that the activity concentration $\left(A_{\text {cons }}\right)$ of the blood does not changed during one heart cycle the EF could then be calculated following:

$$
\begin{aligned}
\mathrm{EF} & =\frac{\mathrm{EDV}-\mathrm{ESV}}{\mathrm{EDV}} \times 100=\frac{\mathrm{EDV}-\mathrm{ESV}}{\mathrm{EDV}} \times \frac{A_{\text {cons }}}{A_{\text {cons }}} \times 100 \\
& =\frac{A_{\text {total EDV }}-A_{\text {total ESV }}}{A_{\text {total EDV }}} \times 100
\end{aligned}
$$

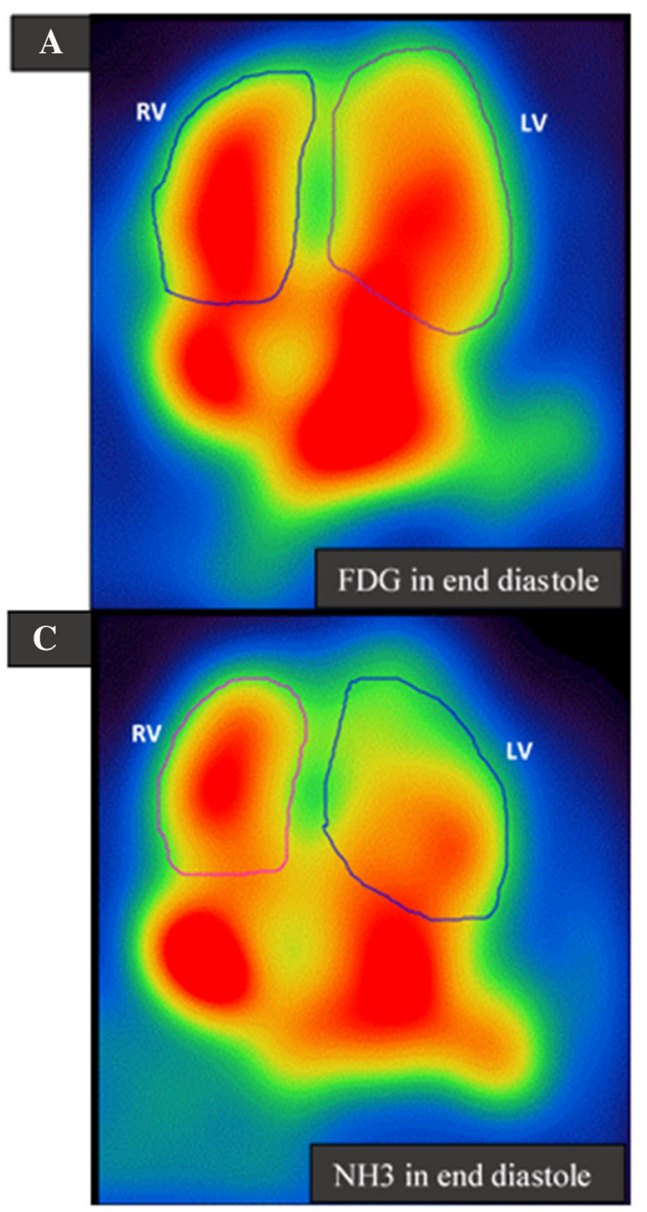

The entire manual segmentation procedure was applied for each patient and for both FDG (Figure 1A, B) and $\mathrm{NH}_{3}$ (Figure 1C, D).

\section{Early- and Late-Phase Left Ventricular Function and Volumes}

Quantitative analysis of both FDG and $\mathrm{NH}_{3}$ PET data for obtaining the left ventricular parameters such as end-systolic volume (LVESV), end-diastolic volume (LVEDV), and ejection fraction (LVEF) from early(EP) and late-phase (LP), i.e., myocardial-uptake, images was conducted using the software QGS ${ }^{\circledR}$ (Version 2013.3, Cedars-Sinai Medical Center, Los Angeles, USA). Applying count-based calculations and automated delineation of myocardium, the software has measured the myocardial EF from ESV and EDV for the

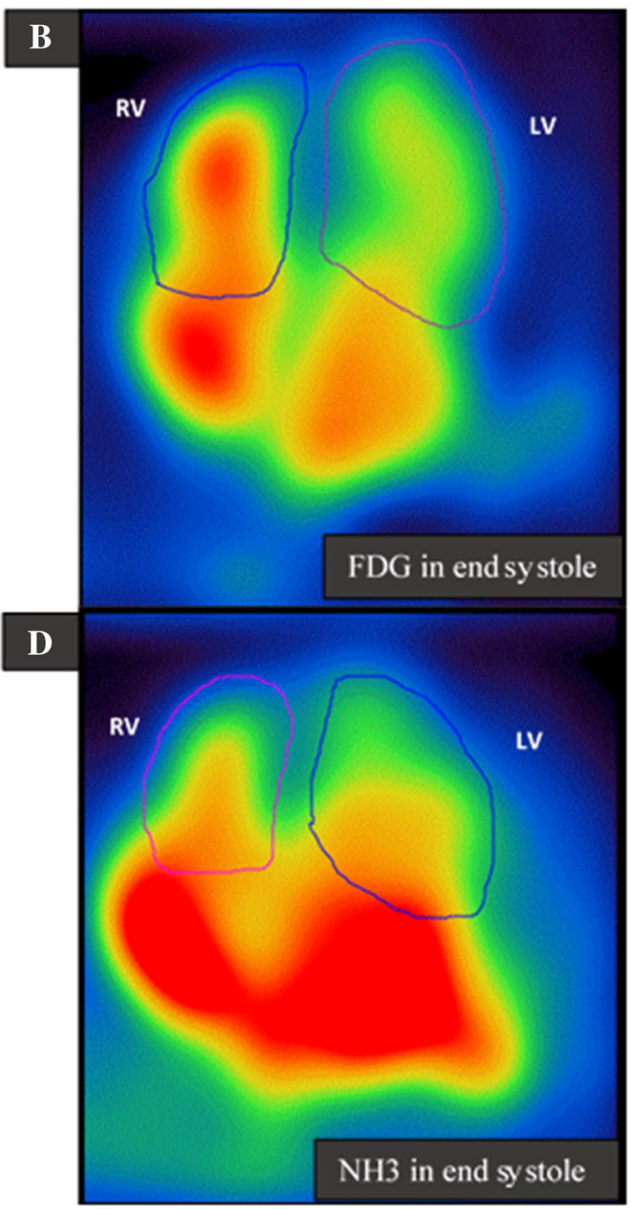

Figure 1. Manual delineation of the right (RV) and left ventricle (LV) from the EDV frames for estimation of first-pass ejection fraction of the left and right ventricle in a 74-year patient with ischemic coronary heart disease. (A) FDG in end-diastolic (ED) and (B) in end-systolic (ES) phase. (C) $\mathrm{NH}_{3}$ in ED and (D) in ES. 


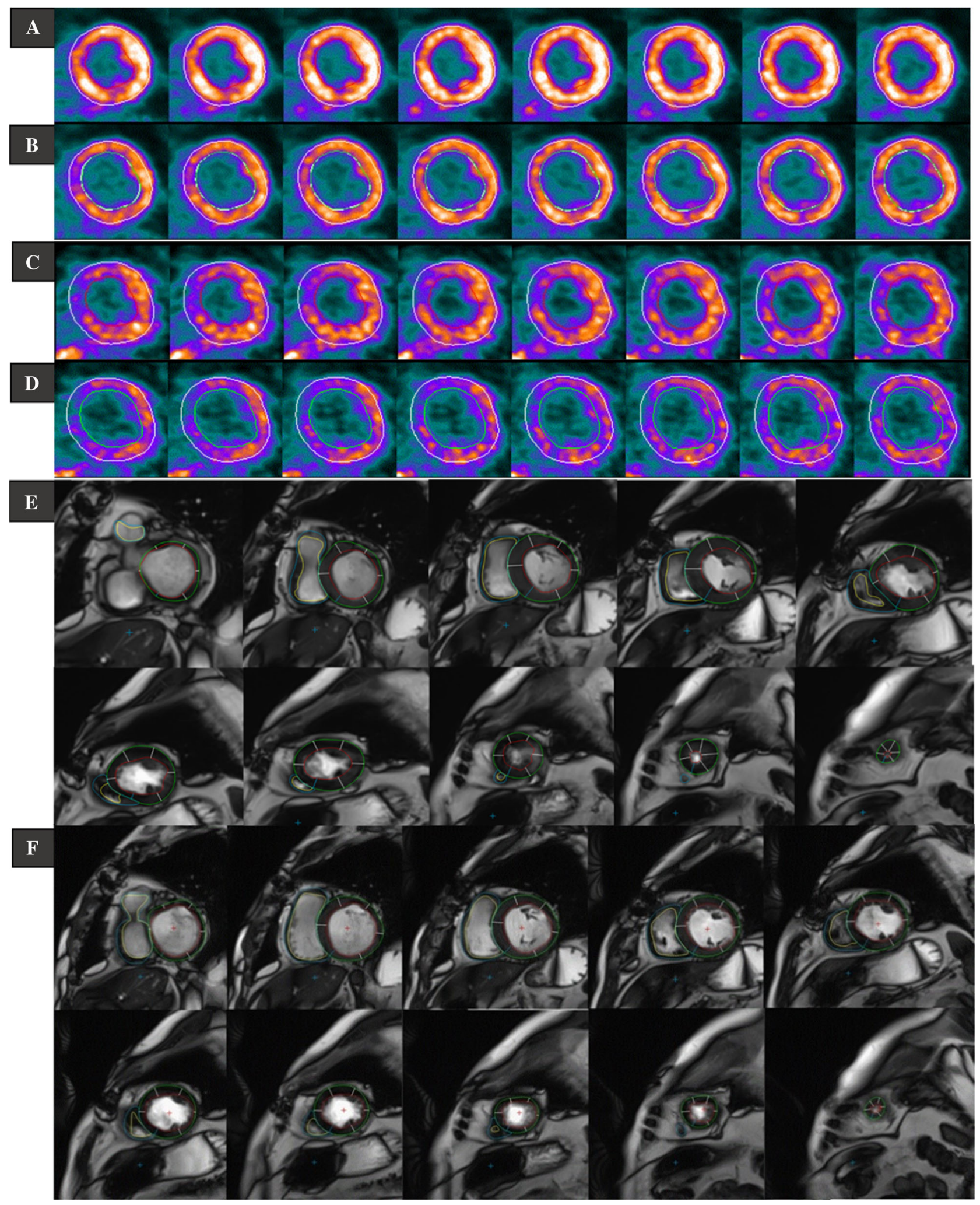

Figure 2. Automated delineation of the left ventricle (LV) for estimation of late-phase cardiac function of left using the software Cedars-Sinai Medical Center: (A) FDG in end systole and (B) in end diastole. (C) $\mathrm{NH}_{3}$ in end systole and (D) in end diastole. Manual delineation of left and right ventricle in CMR of both right ventricle (RV) and LV: (E) in end systole and (F) in end diastole. 

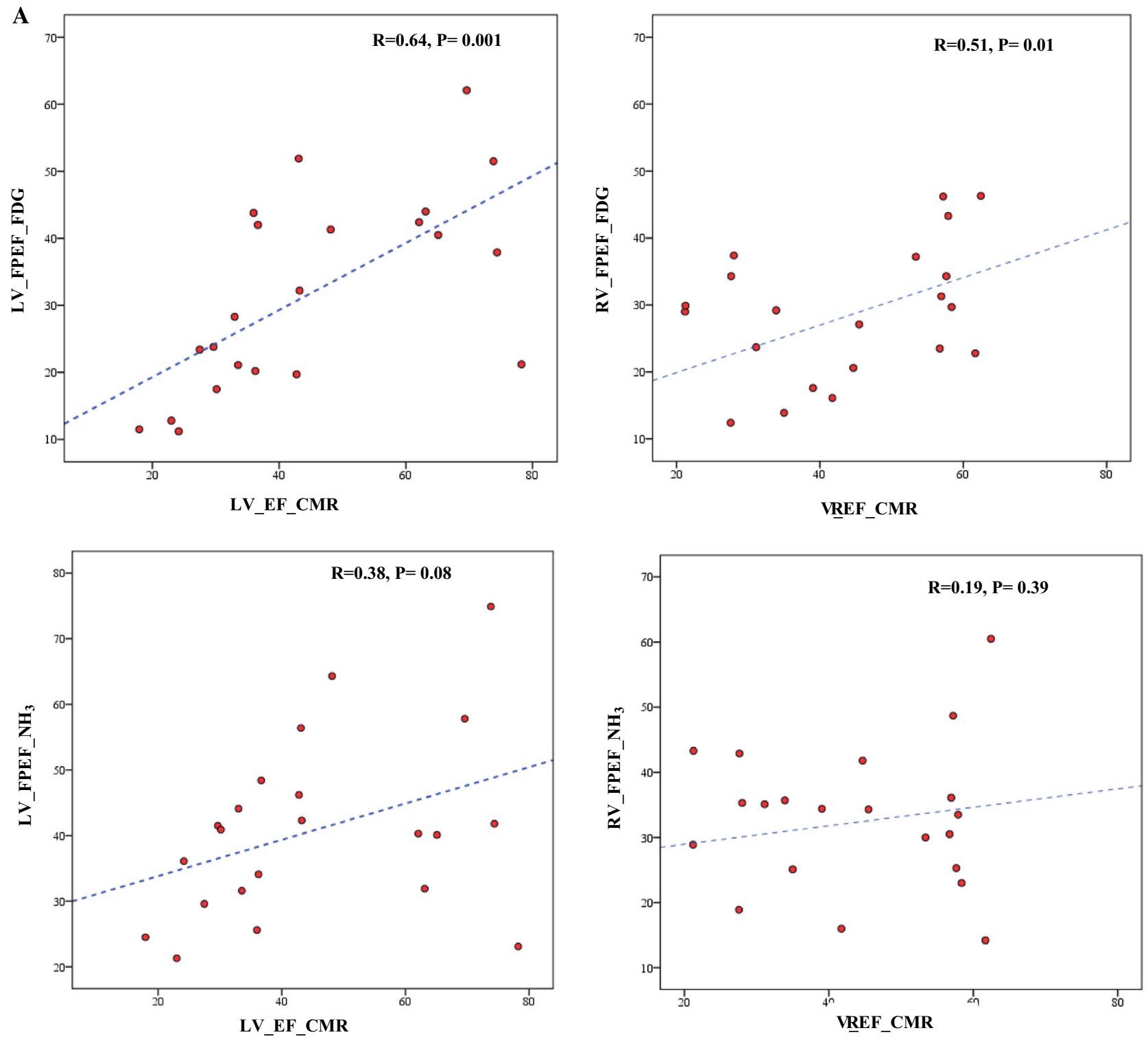

Figure 3. (A) Intra-individual correlation of $\mathrm{LV}$ and RV FPEF acquired from $\mathrm{FDG}$ and $\mathrm{NH}_{3} \mathrm{PET}$ with CMR in an integrated PET/MRI system. LV: left ventricle; RV: right ventricle FPEF: first-pass ejection fraction; CMR: cardiac magnetic resonance imaging. LV and the RV FPEF obtained from FDG PET showed positively moderate $(R=.64, P=.01)$ and weak $(R=.51, P=.01)$ correlations with that received from CMR. No significant correlations were observed between LV and RV FPEF from $\mathrm{NH}_{3}$ PET and CMR. (B) Intra-individual agreements with Bland Altmann analysis of LV and $\mathrm{RV}$ FPEF acquired from FDG and $\mathrm{NH}_{3}$ PET with CMR in an integrated PET/MRI system. $L V$, left ventricle; $R V$, right ventricle FPEF: first-pass ejection fraction; $C M R$, cardiac magnetic resonance imaging. Bland Altmann analysis shows a higher bias of about $14 \% \mathrm{EF}$ (EF unit) for CMR compared to FDG PET and about $10 \%$ higher EF compared to $\mathrm{NH}_{3}$ PET.

left ventricle automatically of FDG PET (Figure 2A, B) and of $\mathrm{NH}_{3}$ PET (Figure 2C, D). For this reason, dynamic ECG-gated images from EP (first 2 to 10 minutes) in 8 and 16 gates and LP (last 10 minutes) of both PET examinations were reconstructed, reoriented, and post-filtered.

\section{CMR Left and Right Ventricular Function and Volumes}

The quantitative analysis of CMR from DICOM images for acquiring LVESV, LVEDV, LVEF and RVEF was achieved by an experienced CMR radiologist according to the MR guidelines of cardiovascular MRI 

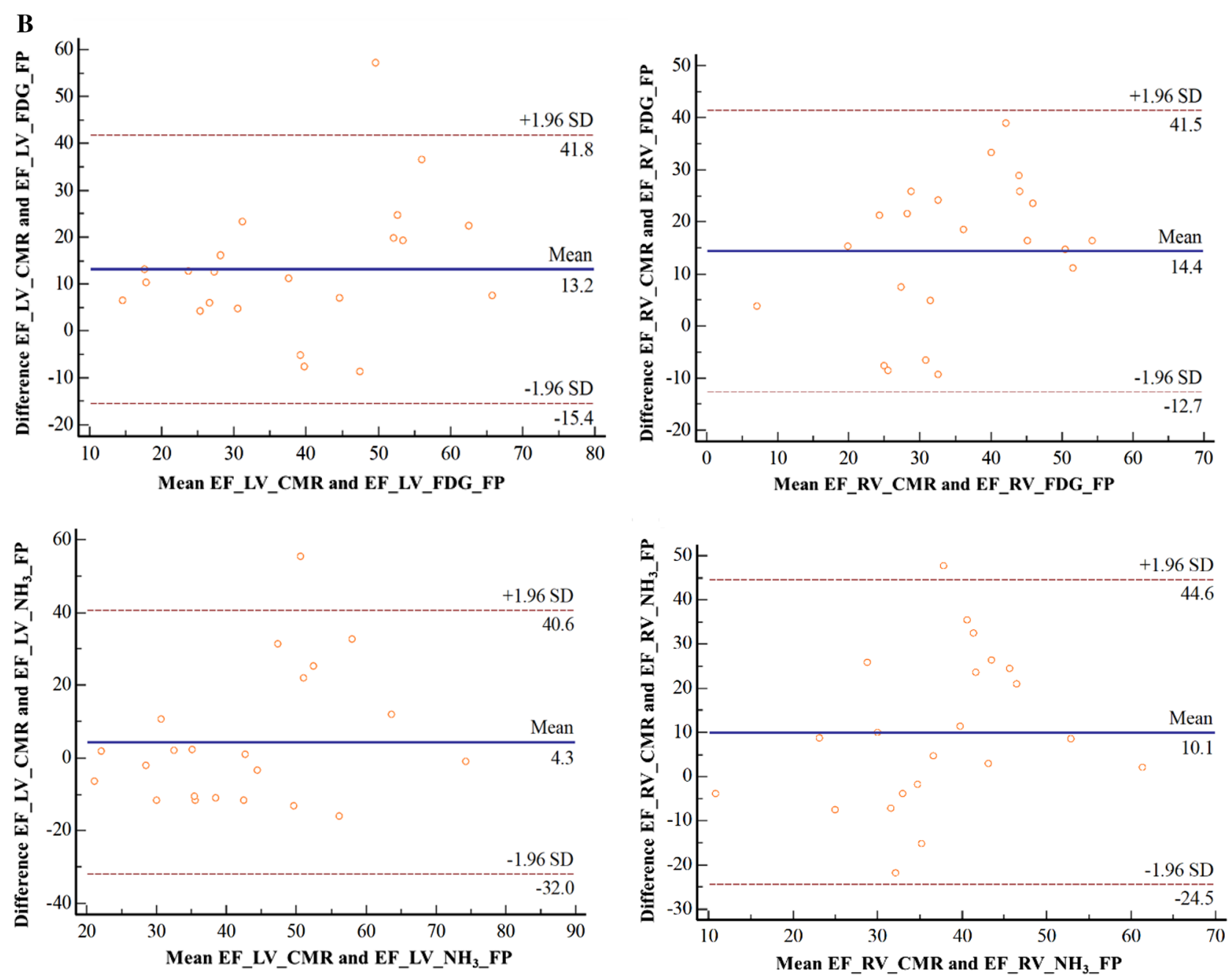

Figure 3. continued.

image interpretations. ${ }^{17}$ All CMR parameters were then post processed using a dedicated cardiac postprocessing software (QMass ${ }^{\circledR}$, MEDIS, Leiden, Netherlands). RVand LVEDV were defined as to have the largest RV and LV blood volume, respectively. The RV- and LVESV were outlined with the smallest RV and LV blood volume, respectively (Fig 2E, F).

\section{Statistical Analysis}

The IBM SPSS Software version 24.0 was used for all data entry and analysis. All obtained data were tested for normality of distribution using the KolmogorovSmirnov test. Parameters that showed a normal distribution were presented in mean \pm standard deviation
(SD). Not normally distributed parameters, if available, were presented in median \pm (minimal - maximal) range and were $\log _{10}$-transformed for analysis. Categorical variables were shown in percentages and number of recorded cases, as stated. Pearson's correlations coefficient was used to study correlations of PET (FDG and $\mathrm{NH}_{3}$ ) parameters with CMR parameters, which were considered as gold standard. Hence, a correlation coefficient $(R)$ of $\geq .5$ and $<.6$ was considered weak, a $R \geq$ .6 and $<.8$ was considered moderate and a $R \geq .8$ was considered strong. ${ }^{18}$ Furthermore, we performed a Bland-Altman plot analysis between PET and CMR concerning FP parameters to additionally show the agreement between the two devices. For all statistical analysis, a $P$-value of $<.05$ was considered statistically significant. 

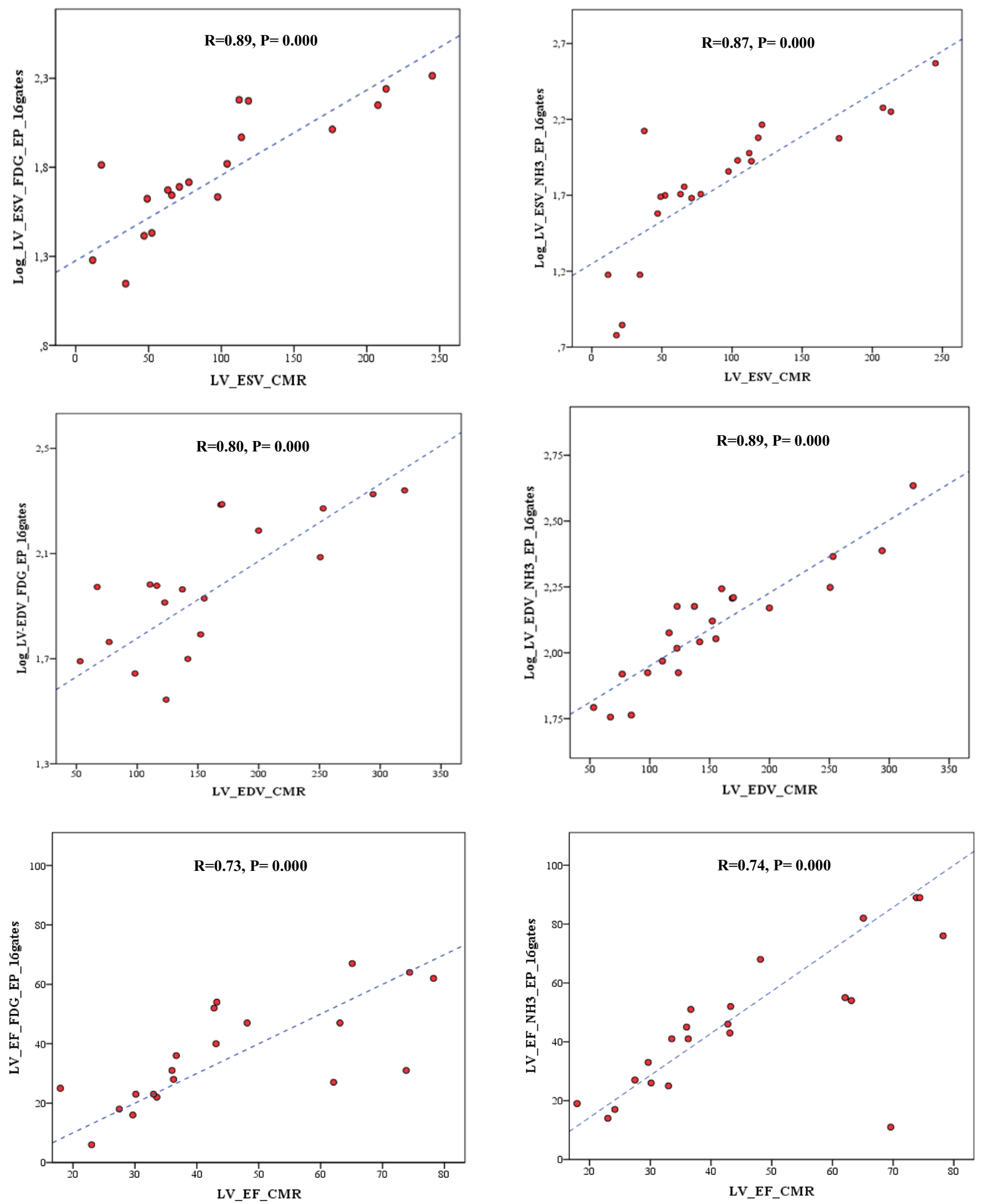
4Figure 4. Intra-individual correlations of LVESV, LVEDV, and LVEF acquired from EP of dynamic FDG and NH3 PET in 16 gates as compared to CMR in an integrated PET/MRI system. $L V$, left ventricle; $R V$, right ventricle; $E S V$, endsystolic volume; $E P$, early-phase; $E D V$, end-diastolic volume; $E F$, ejection fraction; $C M R$, cardiac magnetic resonance imaging. All LV parameters moderately to strongly correlated to CMR when they acquired from EP FDG and $\mathrm{NH}_{3}$ in 16 gates (all $R>.7$ and $P=.000$ ).

\section{RESULTS}

Twenty-two consecutive patients (77\% male, aged $69 \pm 9$ years, body mass index $27.3 \pm 4.6 \mathrm{~kg} / \mathrm{m}^{2}$ ), were eligible for evaluation and the clinical characteristics of these patients and their cardiovascular risk factors are shown in Table 1. Fourteen of 22 patients (64\%) presented with CHD and $6(36 \%)$ suffered from ischemic cardiomyopathy. Mean $\pm \mathrm{SD}$ of blood glucose prior the FDG injection was $124 \pm 33 \mathrm{mg} / \mathrm{dL}$ and the injected $\mathrm{FDG}$ and $\mathrm{NH}_{3}$ activities were $334 \pm 35$ and 816 $\pm 95 \mathrm{MBq}$, respectively. The mean of the heart rate of these patients was $68 \pm 12$ beats/minute. Seven patients (32\%) had type 2 diabetes mellitus, merely 1 (14\%) was insulin-dependent and $6(86 \%)$ were treated with only oral antidiabetic therapies, mostly metformin. $77 \%$ of the evaluated patients presented with arterial hypertension, $64 \%$ with hyperlipidemia, and $14 \%$ were active smokers. Approximately $46 \%$ of the patients presented with at least one coronary stent and $32 \%$ with a coronary bypass graft. $23 \%$ of patients had prior myocardial infarction.

\section{Functional Parameters Acquired from FP FDG and $\mathrm{NH}_{3}$ PET and from CMR}

The RV and LV FPEF obtained from FDG and $\mathrm{NH}_{3}$ were $28 \% \pm 11$ and $32 \% \pm 15$ as well as $32 \% \pm 11$ and $41 \% \pm 14$, respectively. The RVEF and the LVEF received from the CMR were $42 \% \pm 16$ and $45 \% \pm 19$, respectively (Table 2 ).

\section{Functional Parameters Acquired from Early- and Late-Phase FDG and $\mathrm{NH}_{3}$ PET and from CMR}

As presented in Table 3, the LVESV as well as LVEDV and LVEF acquired from EP dynamic FDG in 8 and 16 gates were [71 ( 15 to $213 \mathrm{~mL}$ ), 98 (16 to 241 $\mathrm{mL}$ ) and $32 \pm 17 \%$ ] and [50 (17 to $206 \mathrm{~mL}$ ), 93 (13 to $219 \mathrm{~mL}$ ), and $36 \pm 17 \%$ ], respectively, while LVESV, LVEDV and LVEF acquired from LP dynamic FDG
Figure 5. Intra-individual correlations of LVESV, LVEDV, and LVEF acquired from LP of dynamic FDG and $\mathrm{NH}_{3}$ PET as compared to CMR in an integrated PET/MRI system. $L V$, left ventricle; $R V$, right ventricle; $E S V$, end-systolic volume; $L P$, late-phase; $E D V$, end-diastolic volume; $E F$, ejection fraction; $C M R$, cardiac magnetic resonance imaging. All LV parameters acquired from LP FDG and NH3 correlated good to strongly positive with those received from CMR (all $R>.7$, and $P<$ $.001)$, except EDV from $\mathrm{LP} \mathrm{NH}_{3}$ weakly correlated to the EDV of CMR $(R=.54, P<.05)$.

were $85 \pm 63 \mathrm{~mL}, 138 \pm 63 \mathrm{~mL}$, and $47 \pm 19 \%$, respectively.

Moreover, the LVESV, LVEDV and LVEF measured from EP dynamic $\mathrm{NH}_{3}$ in 8 and 16 gates were [60 (19 to $360 \mathrm{~mL}$ ), 109 (56 to $384 \mathrm{~mL}$ ), and $41 \pm 22 \%$ ] and [54 (16 to $371 \mathrm{~mL}), 116$ (57 to $431 \mathrm{~mL}$ ), and $46 \pm 24 \%$ ], respectively and from $\mathrm{LP}$ dynamic $\mathrm{NH}_{3}$ were $79 \pm 56$ $\mathrm{mL}, 137 \pm 63 \mathrm{~mL}$, and $47 \pm 20 \%$, respectively (Table 3 ).

The LVESV, LVEDV and LVEF acquired from CMR during the integrated PET/MRI examination were $93 \pm 66 \mathrm{~mL}, 153 \pm 71 \mathrm{~mL}$ and $45 \pm 19 \%$, respectively, all in Table 3.

\section{LV and RV FPEF acquired from FDG and $\mathrm{NH}_{3}$ PET compared to CMR}

Although results revealed significant differences between EF of CMR and FPEF of both FDG and NH3 ( $P$ $<.001$ ), the LV and the RV FPEF obtained from FDG PET showed positively moderate and weak correlations with the LVEF and RVEF received from CMR $(R=.64$, $P=.01)$ and $(R=.51, P=.01)$ respectively. Nevertheless, no significant correlations were observed between $\mathrm{LV}$ and RV FPEF obtained from NH3 PET scan as compared to LVEF and RVEF from CMR, all Fig 3A. The Bland Altmann analysis for the agreement between the PET and CMR concerning these parameters showed a higher bias of about 14\% EF (EF unit) for CMR compared to FDG PET and of about only $10 \%$ higher EF compared to $\mathrm{NH}_{3}$ PET (Fig 3B).

\section{Parameters from 8 and 16 Gates of EP Dynamic FDG and $\mathrm{NH}_{3}$ Compared to $\mathrm{CMR}$}

We observed significant differences between CMR and all LV parameters measured from early 2 to 10 minutes of FDG PET in 8 and 16 gates (all $P<.01$ ). Furthermore, there were significant differences between CMR and LV parameters from early 2 to 10 minutes $\mathrm{NH}_{3}$ PET in 8 and 16 gates $(P<.001)$, except for ESV in 16 gates and $\mathrm{EF}$ values in both 8 and 16 gates. 

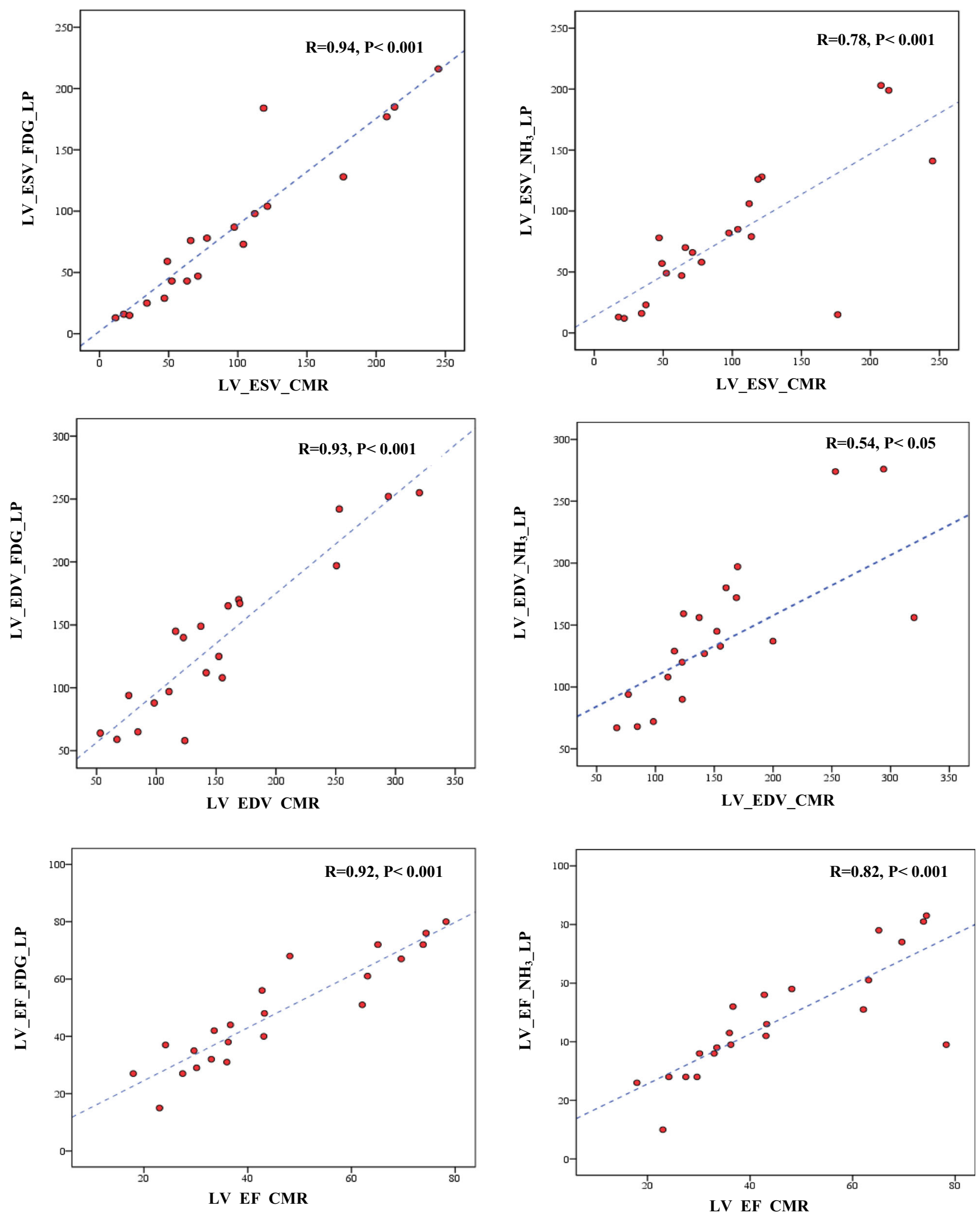
Table 1. Clinical characteristics of study participants $(N=22)$

\begin{tabular}{|c|c|}
\hline Parameters & Values \\
\hline Patients (N) & 22 \\
\hline Male (N) (\%) & (17) $77 \%$ \\
\hline Female (N) (\%) & (5) $23 \%$ \\
\hline Age $($ mean $\pm S D)$ year & $69 \pm 9$ \\
\hline \multicolumn{2}{|l|}{ Underlying cardiac diseases } \\
\hline $\mathrm{CHD}(\mathrm{N})(\%)$ & (14) 64 \\
\hline ICMP (N) (\%) & (8) 36 \\
\hline Height, mean \pm SD $(\mathrm{cm})$ & $175 \pm 10$ \\
\hline Weight, mean \pm SD $(\mathrm{kg})$ & $84 \pm 17$ \\
\hline Body mass index, mean $\pm \mathrm{SD}\left(\mathrm{kg} / \mathrm{m}^{2}\right)$ & $27.3 \pm 4.6$ \\
\hline $\mathrm{NH}_{3}$, mean $\pm \mathrm{SD}(\mathrm{MBq})$ & $816 \pm 95$ \\
\hline $\mathrm{FDG}$, mean $\pm \mathrm{SD}(\mathrm{MBq})$ & $334 \pm 35$ \\
\hline Blood glucose pre FDG injection, mean \pm SD $(\mathrm{mg} / \mathrm{dl})$ & $124 \pm 33$ \\
\hline Heart rate beats/minutes & $68 \pm 12$ \\
\hline \multicolumn{2}{|l|}{ Lipid parameters (mean \pm SD) } \\
\hline Triglyceride & $162 \pm 68$ \\
\hline Total cholesterol & $149.5 \pm 55$ \\
\hline LDL & $43 \pm 12.9$ \\
\hline $\mathrm{HDL}^{*}$ & $81.6(46-132)$ \\
\hline Type 2 diabetes mellitus (N) (\%) & (7) 32 \\
\hline Insulin-dependent (N) (\%) & (1) 14 \\
\hline On OADs $(\mathrm{N})(\%)$ & (6) 86 \\
\hline \multicolumn{2}{|l|}{ Cardiovascular risk factors } \\
\hline Arterial hypertension (N) (\%) & (17) 77.3 \\
\hline Hyperlipidemia (N) (\%) & (14) 63.6 \\
\hline \multicolumn{2}{|l|}{ Smoking status (N) (\%) } \\
\hline Current & (3) 13.6 \\
\hline Former & (6) 27.3 \\
\hline Never & (7) 31.8 \\
\hline Unknown & (6) 27.7 \\
\hline \multicolumn{2}{|l|}{ Previous history (N) (\%) } \\
\hline Coronary stents & (10) 45.5 \\
\hline Bypass operation & (7) 31.8 \\
\hline Myocardial infarction & (5) 22.7 \\
\hline
\end{tabular}

$H D L$ high density lipoprotein; ICMP ischemic cardiomyopathy; $L D L$ low density lipoprotein; $O A D s$ oral antidiabetics

${ }^{*}$ Not normally distributed and presented in median \pm (minimal - maximal) range

However, all the LV parameters moderately to strongly correlated to CMR when they acquired from EP FDG and $\mathrm{NH}_{3}$ in 16 gates (all $R>.7$ and $P=.000$ ), Figure 4 .

\section{Parameters from LP Dynamic FDG and $\mathrm{NH}_{3}$ PET Compared to CMR}

There were no significant differences between LV parameters from CMR and LP dynamic from both FDG and $\mathrm{NH}_{3} \mathrm{PET}$, except for EDV obtained from FDG. In total, all LV parameters that acquired from LP dynamic
FDG and $\mathrm{NH}_{3}$ correlated good to strongly positive with those received from CMR (all $R>.7$, and $P<.001$ ), except EDV from $\mathrm{LP} \mathrm{NH}_{3}$ weakly correlated to the EDV of CMR $(R=.54, P<.05)$, all shown in Figure 5.

\section{DISCUSSION}

In a cohort of patients with advanced CHD, we used an integrated PET/MRI system to intra-individually compare cardiac functional parameters obtained from dynamic ECG-gated FDG and $\mathrm{NH}_{3}$ PET scans with 3- 
Table 2. Ejection fraction of the right and left ventricle assessed with dynamic gated $\mathrm{FDG}$ and $\mathrm{NH}_{3} \mathrm{PET}$ both in first 2 minutes of image acquisition and with CMR in an integrated PET/MRI $(\mathrm{N}=22)$

\section{First-pass (0-2 minutes)}

Image modality

Right ventricle

Left ventricle

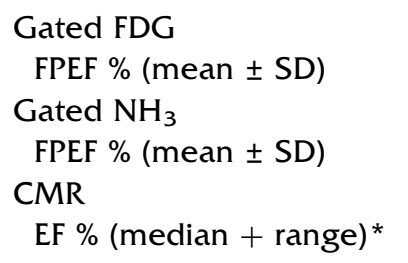

$\mathrm{EF} \%$ (median + range)*

$28 \pm 11^{\#}$

$32 \pm 15^{\#}$

$32 \pm 11^{\S}$

$41 \pm 14$

* Not normass ejection fraction; $S D$, standard deviation

"Significant differences between CMR and FDG parameters $(P<.001)$

${ }^{s}$ Significant differences between $\mathrm{CMR}$ and $\mathrm{NH}_{3}$ parameters $(P<.001)$

Table 3. Parameters of left ventricular function assessed with QGS software obtained from early (8 and 16 gated) and late imaging phase of FDG and $\mathrm{NH}_{3}$ PET scan and from CMR in an integrated PET/ MRI $(\mathrm{N}=22)$

\section{Image modality}

Parameters of the left ventricular function

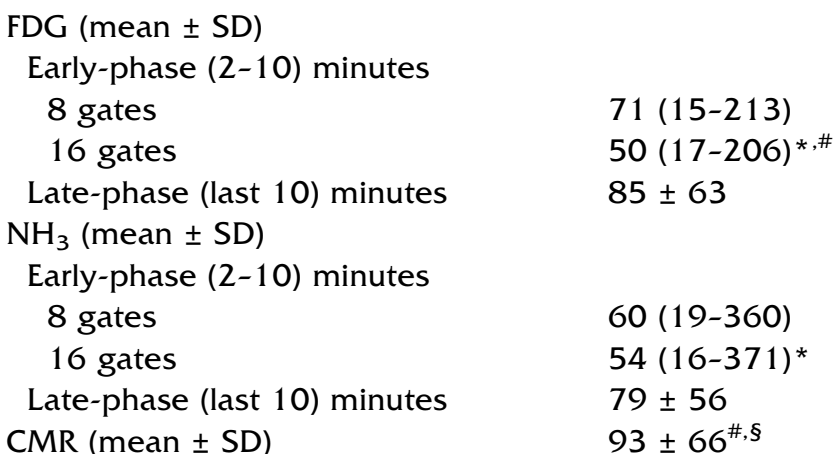

$E S V$, end-systolic volume; $E D V$, end-diastolic volume; $E F$, ejection fraction; $S D$, standard deviation

*Not normally distributed and presented in median + range (minimal - maximal)

\#Significant differences between CMR and FDG parameters $(P<.01)$

${ }^{\S}$ Significant differences between $\mathrm{CMR}$ and $\mathrm{NH}_{3}$ parameters $(P<.001)$

Tesla-MRI functional parameters as a standard reference.

While the first-pass FDG data showed significant correlations between LVFPEF and RVFPEF with those gained from $\mathrm{CMR}$, this was not the case for $\mathrm{NH}_{3}$. However, a systemic underestimation of the EF was found for both FDG (14\%) and $\mathrm{NH}_{3}(10 \%)$. In this respect, Todica et al. previously acquired ECG-gated
FDG PET examinations in seven healthy rats and found a good correlation between LV FPEF from FDG PET and LVEF values from CMR. ${ }^{9}$ In their study, functional parameters were calculated with the QBS $^{\circledR}$ software (Cedars-Sinai, Los Angeles, USA) for evaluating gated FDG blood-pool PET in the studied rats. Similarly, in 24 oncological patients, Bouallègue et al. could recently show possibility of assessing cardiac function from the 
gated FDG PET studies. ${ }^{11}$ They revealed a very good agreement between the results of LV FPEF obtained from gated FDG PET and those acquired from SPECT studies with equilibrium radionuclide angiography, where LV FPEF determined with an automatic segmentation software. Indeed, our current study is the first clinical research demonstrating the feasibility of calculating LV FPEF and RV FPEF from dynamic FDG and $\mathrm{NH}_{3}$ PET scans in patients with CHD. The disparities in correlations of FDG and $\mathrm{NH}_{3}$ PET with CMR observed in this study might be caused by the fact that both these tracers have different uptake-mechanisms in the myocardium. Due to the more rapid first-pass uptake of $\mathrm{NH}_{3}$ into the myocardium, ${ }^{19}$ thus, the assumption that the activity in the myocardium can be neglected during the first-pass to be able to calculate the EF form an extended VOI around the ventricle may not be entirely correct and particularly with $\mathrm{NH}_{3}$. Moreover, the different count statistics within the $\mathrm{NH}_{3}$ images may affected the manual ventricle delineation. Furthermore, the relatively high activity concentration of $\mathrm{NH}_{3}(816 \pm 95 \mathrm{MBq})$ applied in our studied cohort should be taken into consideration, as this might increase the number of the detected random events and influencing the deadtime in the PET/MR detector system and affecting the reading of $\mathrm{NH}_{3}$ images. ${ }^{20}$ Nevertheless, the significant association of right and left ventricular FPEF from dynamic ECG-gated FDG examinations with that from CMR might enable concomitant assessment of cardiac function while performing a whole-body oncological FDG PET/MRI or even PET/CT. Basically, FDG PET scans using PET/CT or PET/MRT systems are frequently performed worldwide in a wide range of diagnostic areas, ${ }^{21}$ especially in oncology. Performing a dynamic blood-pool PET acquisition over the heart would allow the first-pass acquisition of cardiac functional parameters, which are important to early detect organ damage under the effect of cardiotoxic oncological therapies.

Moreover, results of the early- and late-phase analyses showed overall good to strong correlations between the left ventricular functional parameters such as LVESV, LVEDV, and LVEF acquired from both $\mathrm{NH}_{3}$ and FDG PET scans with that of CMR. In fact, because $\mathrm{NH}_{3}$ is a tracer commonly used to determine myocardial blood flow, ${ }^{22}$ studies that have directly compared cardiac functional parameters obtained from $\mathrm{NH}_{3} \mathrm{PET}$ with those acquired from CMR are very limited. In a very recent $3 \mathrm{~T}$ hybrid PET/MRI study, Nazir et al. could rather demonstrate a feasible quantification of myocardial blood flow with good to moderate agreement between PET and CMR in a phantom and five healthy volunteers undergoing adenosine stress, where $\mathrm{NH}_{3}$ and gadolinium were administered simultaneously. ${ }^{23}$ In an earlier study, Okazawa et al. showed in a subgroup of the examined participants with cardiovascular diseases a good agreement of the LVEF from $\mathrm{NH}_{3}$ PET with that obtained from left ventriculography. ${ }^{24}$ There are, albeit, some studies that found precise determination of cardiac function from ECG-gated $\mathrm{NH}_{3}$, when FDG cardiac function values were considered as gold standard. ${ }^{25,26}$

Furthermore, results from previous preclinical and human studies acquired with separate PET and CMR scanners using the late-phase FDG PET are in one line with the present study. ${ }^{27-29}$ Stegger et al. revealed in a preclinical study with 33 mice, mostly with occlusions of at least one coronary artery, a good agreement of cardiac function measures from FDG PET with that of CMR. ${ }^{30}$ Todica et al. were able to prove this agreement between FDG PET and CMR in healthy mice as well. ${ }^{9}$ Similar correlations were found for the comparison between $\left[{ }^{68} \mathrm{Ga}\right.$ ]Albumin PET, an experimental bloodpool marker, and CMR. ${ }^{31}$ Furthermore, excellent correlations for $\mathrm{LV}$ myocardial function were observed between FDG and CMR in a human study by Slart et al. that included 38 patients with chronic coronary heart diseases received separately gated FDG PET and $\mathrm{CMR}^{7}$ and in a study by $\mathrm{Li}$ et al. with patients with heart failure. ${ }^{8}$ Altogether, these results reveal that left ventricular parameters obtained from late-phase $\mathrm{NH}_{3}$ PET and right and left ventricular parameters acquired from first-pass and late-phase gated FDG PET examination can accurately be used to evaluate cardiac function.

Nonetheless, the retrospective design and the small sample size of the included cardiac PET/MRI scans, among them data of $32 \%$ type 2 diabetic patients, might limit the results of this study. Moreover, the used activity of about $800 \mathrm{MBq}$ was above the peak noise equivalent count rate (NEC) of the system. ${ }^{32}$ Therefore, the absolute values of the activity concentrations measured during the first-pass are biased, as we cannot assume that all corrections (e.g., deadtime) work well at these high activity levels. However, we expect that this bias is a constant multiplicative factor for the 8 gates used for the calculation of the EF from the fist-pass images. Therefore, the bias cancels out in the formula $(\mathrm{EDV} \times$ bias $-\mathrm{ESV} \times$ bias $) / \mathrm{EDV} \times$ bias $=(\mathrm{EDV}-$ $\mathrm{ESV}) \times$ bias $/ \mathrm{EDV} \times$ bias $=(\mathrm{EDV}-\mathrm{ESV}) / \mathrm{EDV}$ and an eventual bias should not have any effect on the calculation of the EF. Additionally, results of this intraindividual comparison might have been influenced by the reader-dependent delineation of the ventricles of FP PET and particularly the RV, which is already considered a challenge, ${ }^{33}$ as an inaccurate ROI drawing might lead to inappropriate determination of the RV and LV contours in PET and MRI. Therefore, manual delineations using an automatic or semi-automatic delineating technique as that used in QBS ${ }^{\circledR}$ software might reduce reader dependence and further improve FP 
PET measures correlations with that of CMR. Finally, even it is unlikely that the blood-pool phase/early-phase of FDG distribution is significantly affected by different protocols and patient preparation, the transfer of our results into an oncological setting has to be made with caution.

\section{NEW KNOWLEDGE GAINED}

Based on the results of this study, deriving reliable LV and RV functional parameters from various examination phases of the dynamic $\mathrm{NH}_{3}$ and FDG is feasible and could potentially be estimated from other, mostly oncological routine scans when dynamic list-mode FDG PET acquisition is performed.

\section{CONCLUSIONS}

Findings of this intra-individual PET/MRI analysis in patients with advanced CHD demonstrated the feasibility to calculate left and right ventricular function from several phases of dynamic $\mathrm{NH}_{3}$ and FDG PET scans using the first 2 as well as early 2 to 10 and late 10 minutes of PET list-mode data. There was a good agreement between the left ventricular ejection fraction calculated from the first-pass FDG PET acquisition and CMR, which could potentially open new perspectives for dynamic PET acquisitions in the oncological field or novel total body PET applications.

\section{Acknowledgment}

We thank Kaspar Hoeschl, M.Sc., from Vienna University of Technology as well as Julia Kesselbacher and Benedikt Schmiedinger from our PET/MRI section for reconstructions and data processing of FDG and $\mathrm{NH}_{3}$ PET images.

\section{Funding} Vienna.

Open access funding provided by Medical University of

\section{Disclosures}

Sazan Rasul, Dietrich Beitzke, Tim Wollenweber, Ivo Rausch, Martin Lyngby Lassen, Marie Elisabeth Stelzmüller, Markus Mitterhauser, Verena Pichler, Thomas Beyer, Christian Loewe, and Marcus Hacker have no conflict of interest or any disclosures relevant to this paper.

\section{Open Access}

This article is licensed under a Creative Commons Attribution 4.0 International License, which permits use, sharing, adaptation, distribution and reproduction in any medium or format, as long as you give appropriate credit to the original author(s) and the source, provide a link to the Creative Commons licence, and indicate if changes were made. The images or other third party material in this article are included in the article's Creative Commons licence, unless indicated otherwise in a credit line to the material. If material is not included in the article's Creative Commons licence and your intended use is not permitted by statutory regulation or exceeds the permitted use, you will need to obtain permission directly from the copyright holder. To view a copy of this licence, visit http://creativecommons.org/licenses/by/4.0/.

\section{References}

1. Roth GA, Johnson C, Abajobir A, Abd-Allah F, Abera SF, Abyu $\mathrm{G}$, et al. Global, regional, and national burden of cardiovascular diseases for 10 causes, 1990 to 2015. J Am Coll Cardiol. 2017;70:1-25.

2. Nensa F, Bamberg F, Rischpler C, Menezes L, Poeppel TD, la Fougere C, et al. Hybrid cardiac imaging using PET/MRI: a joint position statement by the European Society of Cardiovascular Radiology (ESCR) and the European Association of Nuclear Medicine (EANM). Eur Radiol. 2018;28:4086-101.

3. Ghosh N, Rimoldi OE, Beanlands RS, Camici PG. Assessment of myocardial ischaemia and viability: role of positron emission tomography. Eur Heart J. 2010;31:2984-95.

4. Kostakoglu L, Agress H, Jr., Goldsmith SJ. Clinical role of FDG PET in evaluation of cancer patients. Radiographics. 2003;23:31540; quiz 533.

5. Broder H, Gottlieb RA, Lepor NE. Chemotherapy and cardiotoxicity. Rev Cardiovasc Med. 2008;9:75-83.

6. Fraum TJ, Fowler KJ, McConathy J. PET/MRI: Emerging clinical applications in oncology. Acad Radiol. 2016;23:220-36.

7. Slart RH, Bax JJ, de Jong RM, de Boer J, Lamb HJ, Mook PH, et al. Comparison of gated PET with MRI for evaluation of left ventricular function in patients with coronary artery disease. $\mathrm{J}$ Nucl Med. 2004;45:176-82.

8. Li Y, Wang L, Zhao SH, He ZX, Wang DY, Guo F, et al. Gated F18 FDG PET for assessment of left ventricular volumes and ejection fraction using QGS and 4D-MSPECT in patients with heart failure: A comparison with cardiac MRI. PLoS ONE. 2014;9:e80227.

9. Todica A, Boning G, Lehner S, Weidl E, Cumming P, Wangler C, et al. Positron emission tomography in the assessment of left ventricular function in healthy rats: a comparison of four imaging methods. J Nucl Cardiol. 2013;20:262-74.

10. Ben Bouallegue F, Mariano-Goulart D, Agostini D, Manrique A. Feasibility of biventricular volume and function assessment using first-pass gated (15)O-water PET. EJNMMI Res. 2018;8:92.

11. Ben Bouallegue F, Maimoun L, Kucharczak F, Le Fur P, Vauchot F, Hay B, et al. Left ventricle function assessment using gated first-pass (18)F-FDG PET: Validation against equilibrium radionuclide angiography. J Nucl Cardiol. 2019. https://doi.org/10. 1007/s12350-019-01731-x.

12. Dilsizian V, Bacharach SL, Beanlands RS, Bergmann SR, Delbeke $\mathrm{D}$, Dorbala $\mathrm{S}$, et al. ASNC imaging guidelines/SNMMI procedure standard for positron emission tomography (PET) nuclear cardiology procedures. J Nucl Cardiol. 2016;23:1187-226.

13. Chang SA, Kim RJ. The use of cardiac magnetic resonance in patients with suspected coronary artery disease: A clinical practice perspective. J Cardiovasc Ultrasound. 2016;24:96-103. 
14. Knuuti MJ, Yki-Jarvinen H, Voipio-Pulkki LM, Maki M, Ruotsalainen U, Harkonen R, et al. Enhancement of myocardial [fluorine-18]fluorodeoxyglucose uptake by a nicotinic acid derivative. J Nucl Med. 1994;35:989-98.

15. Beitzke D, Rasul S, Lassen ML, Pichler V, Senn D, Stelzmuller $\mathrm{ME}$, et al. Assessment of myocardial viability in ischemic heart disease by PET/MRI: Comparison of left ventricular perfusion, hibernation, and scar burden. Acad Radiol. 2019;27:188-97.

16. Lassen ML, Beyer T, Berger A, Beitzke D, Rasul S, Buther F, et al. Data-driven, projection-based respiratory motion compensation of PET data for cardiac PET/CT and PET/MR imaging. J Nucl Cardiol. 2019. https://doi.org/10.1007/s12350-019-01613-.

17. Schulz-Menger J, Bluemke DA, Bremerich J, Flamm SD, Fogel MA, Friedrich MG, et al. Standardized image interpretation and post processing in cardiovascular magnetic resonance: Society for Cardiovascular Magnetic Resonance (SCMR) board of trustees task force on standardized post processing. J Cardiovasc Magn Reson. 2013;15:35.

18. Akoglu H. User's guide to correlation coefficients. Turk J Emerg Med. 2018;18:91-3.

19. Machac J. Radiopharmaceuticals for clinical cardiac PET. Imaging. 2007. https://doi.org/10.1007/978-0-387-38295-1_5.

20. O’Doherty J, Chalampalakis Z, Schleyer P, Nazir MS, Chiribiri A, Marsden PK. The effect of high count rates on cardiac perfusion quantification in a simultaneous PET-MR system using a cardiac perfusion phantom. EJNMMI Phys. 2017;4:31.

21. Nensa F, Beiderwellen K, Heusch P, Wetter A. Clinical applications of PET/MRI: current status and future perspectives. Diagn Interv Radiol. 2014;20:438-47.

22. Kuhle WG, Porenta G, Huang SC, Buxton D, Gambhir SS, Hansen $\mathrm{H}$, et al. Quantification of regional myocardial blood flow using $13 \mathrm{~N}$-ammonia and reoriented dynamic positron emission tomographic imaging. Circulation. 1992;86:1004-17.

23. Nazir MS, Gould SM, Milidonis X, Reyes E, Ismail TF, Neji R, et al. Simultaneous (13)N-ammonia and gadolinium first-pass myocardial perfusion with quantitative hybrid PET-MR imaging: A phantom and clinical feasibility study. Eur J Hybrid Imaging. 2019;3:15

24. Okazawa H, Takahashi M, Hata T, Sugimoto K, Kishibe Y, Tsuji T. Quantitative evaluation of myocardial blood flow and ejection fraction with a single dose of (13) $\mathrm{NH}(3)$ and gated PET. J Nucl Med. 2002;43:999-1005.
25. Szymanski MK, Kruizinga S, Tio RA, Willemsen AT, Schafers MA, Stegger L, et al. Use of gated 13N-NH3 micro-PET to examine left ventricular function in rats. Nucl Med Biol. 2012;39:724-9.

26. Khorsand A, Graf S, Eidherr H, Wadsak W, Kletter K, Sochor H, et al. Gated cardiac 13N-NH3 PET for assessment of left ventricular volumes, mass, and ejection fraction: comparison with electrocardiography-gated 18F-FDG PET. J Nucl Med. 2005;46:2009-13.

27. Schaefer WM, Lipke CS, Nowak B, Kaiser HJ, Reinartz P, Buecker A, et al. Validation of QGS and 4D-MSPECT for quantification of left ventricular volumes and ejection fraction from gated 18F-FDG PET: comparison with cardiac MRI. J Nucl Med. 2004;45:74-9.

28. Khorsand A, Graf S, Frank H, Kletter K, Sochor H, Maurer G, et al. Model-based analysis of electrocardiography-gated cardiac (18)F-FDG PET images to assess left ventricular geometry and contractile function. J Nucl Med. 2003;44:1741-6.

29. Higuchi T, Nekolla SG, Jankaukas A, Weber AW, Huisman MC, Reder S, et al. Characterization of normal and infarcted rat myocardium using a combination of small-animal PET and clinical MRI. J Nucl Med. 2007;48:288-94.

30. Stegger L, Heijman E, Schafers KP, Nicolay K, Schafers MA, Strijkers GJ. Quantification of left ventricular volumes and ejection fraction in mice using PET, compared with MRI. J Nucl Med. 2009:50:132-8.

31. Todica A, Brunner S, Boning G, Lehner S, Nekolla SG, Wildgruber $\mathrm{M}$, et al. [68Ga]-albumin-PET in the monitoring of left ventricular function in murine models of ischemic and dilated cardiomyopathy: Comparison with cardiac MRI. Mol Imaging Biol. 2013;15:441-9.

32. Delso G, Furst S, Jakoby B, Ladebeck R, Ganter C, Nekolla SG, et al. Performance measurements of the Siemens mMR integrated whole-body PET/MR scanner. J Nucl Med. 2011;52:1914-22.

33. Tulevski II, Romkes H, Dodge-Khatami A, van der Wall EE, Groenink M, van Veldhuisen DJ, et al. Quantitative assessment of the pressure and volume overloaded right ventricle: imaging is a real challenge. Int J Cardiovasc Imaging. 2002;18:41-51.

Publisher's Note Springer Nature remains neutral with regard to jurisdictional claims in published maps and institutional affiliations. 